\title{
Adaptive Electricity Scheduling in Microgrids
}

\author{
Yingsong Huang, Student Member, IEEE, Shiwen Mao, Senior Member, IEEE, and R. M. Nelms, Fellow, IEEE
}

\begin{abstract}
Microgrid (MG) is a promising component for future smart grid (SG) deployment. The balance of supply and demand of electric energy is one of the most important requirements of MG management. In this paper, we present a novel framework for smart energy management based on the concept of quality-of-service in electricity (QoSE). Specifically, the resident electricity demand is classified into basic usage and quality usage. The basic usage is always guaranteed by the MG, while the quality usage is controlled based on the MG state. The microgrid control center (MGCC) aims to minimize the MG operation cost and maintain the outage probability of quality usage, i.e., QoSE, below a target value, by scheduling electricity among renewable energy resources, energy storage systems, and macrogrid. The problem is formulated as a constrained stochastic programming problem. The Lyapunov optimization technique is then applied to derive an adaptive electricity scheduling algorithm by introducing the QoSE virtual queues and energy storage virtual queues. The proposed algorithm is an online algorithm. We derive several "hard" performance bounds for the proposed algorithm, and evaluate its performance with trace-driven simulations. The simulation results demonstrate the efficacy of the proposed electricity scheduling algorithm.
\end{abstract}

Index Terms-Distributed renewable energy resource, Lyapunov optimization, microgrids, smart grid, stability.

\section{INTRODUCTION}

$\mathbf{S}$ MART GRID (SG) is a modern evolution of the utility generation and delivery system. SG enhances the traditional power grid through computing, communications, networking, and control technologies throughout the processes of electricity generation, transmission, distribution and consumption. The two-way flow of electricity and real-time information is a characteristic feature of SG, which offers many technical benefits and flexibilities to both utility providers and consumers, for balancing supply and demand in a timely fashion and improving energy efficiency and grid stability. According to the U.S. 2009 Recovery Act [2], an SG will replace the traditional system and is expected to save consumer cost and reduce America's dependence on foreign oil. These goals are to be achieved by improving efficiency and spurring the use of renewable energy resources.

Manuscript received December 10, 2012; revised April 12, 2013 and July 21, 2013; accepted September 17, 2013. Date of current version December 24, 2013. This work was supported in part by the U.S. National Science Foundation under Grant CNS-0953513, and through the Broadband Wireless Access and Applications Center (BWAC) Site at Auburn University. This work was presented in part at IEEE INFOCOM 2013, Turin, Italy, Apr. 2013 [1]. Paper no. TSG-00825-2012.

The authors are with the Department of Electrical and Computer Engineering, Auburn University, Auburn, AL 36849-5201 USA (e-mail: yzh0002@tigermail. auburn.edu; smao@ieee.org; nelmsrm@auburn.edu).

Color versions of one or more of the figures in this paper are available online at $\mathrm{http} / /$ ieeexplore.ieee.org.

Digital Object Identifier 10.1109/TSG.2013.2282823
Microgrid (MG) is a promising component for future SG deployment. Due to the increasing deployment of distributed renewable energy resources (DRERs), MG provides a localized cluster of renewable energy generation, storage, distribution and local demand, to achieve reliable and effective energy supply with simplified implementation of SG functionalities [3], [4]. A typical MG architecture consists of DRERs (e.g., wind turbines and solar photovoltaic cells), energy storage systems (ESS), a communication network (e.g., wireless or powerline communications) for information delivery, an MG central controller (MGCC), and local residents. The MGCC controls the MG operation and exchanges information with local residents, ESSs, and DRERs via the information network. There is a single common coupling point with the macrogrid. When disconnected, the MG works in the islanded mode and DRERs and ESSs provide electricity to local residents. When connected, the MG may purchase extra electricity from the macrogrid or sell excess energy back to the market [5].

The balance of electricity demand and supply is one of the most important requirements in MG management. Instead of matching supply to demand, smart energy management matches the demand to the available supply using direct load control or off-peak pricing to achieve more efficient capacity utilization [3]. In this paper, we develop a novel control framework for MG energy management, exploiting the two-way flows of electricity and information. In particular, we consider two types of electricity usage: i) a pre-agreed basic usage that is "hard"-guaranteed, such as basic living usage, and ii) extra elastic quality usage exceeding the pre-agreed level for more comfortable life, such as excessive use of air conditioners or entertainment devices. In practice, residents may set their load priority and preference to obtain the two types of usage [6]. The basic usage should be always satisfied, while the quality usage is controlled by the MGCC according to the grid status, such as DRER generation, ESS storage levels and utility prices. The MGCC may block some quality usage demand if necessary. This can be implemented by incorporating smart meters, smart loads and appliances that can adjust and control their service level through communication flows [5].

To quantify residents' satisfaction level, we define the outage percentage of the quality usage as Quality of Service in Electricity (QoSE), which can be interpreted as the reliability level for the quality usage for residential users [7]. This reliability metric is specified in the service contracts similar to [8] and will be guaranteed by the proposed electricity energy management algorithm. The MGCC adaptively schedules electricity to keep the QoSE below a target level, and dynamically balance the load demand to match the available supply.

In this paper, we investigate the problem of smart energy scheduling by jointly considering renewable energy distribution, ESS management, residential demand management, and 
utility market participation, aiming to minimize the MG operation cost and guarantee the residents' QoSE. The MGCC may serve some quality usage with supplies from the DRERs, ESSs, and macrogrid. On the other hand, the MG can also sell excessive electricity back to the macrogrid to compensate for the energy generation cost. The electricity generated from renewable sources is generally random, due to complex weather conditions, while the electricity demand is also random due to the random consumer behavior, and so do the purchasing and selling prices on the utility market. It is challenging to model the random supply, demand, and price processes for MG management, and it may also be costly to have precise, real-time monitoring of the random processes. Therefore, a simple, low cost, and optimal electricity scheduling scheme that does not rely on any future information of the supply, demand, and price processes would be highly desirable [9], [10].

We tackle the MG electricity scheduling problem with a $L y a-$ punov optimization approach, which is a useful technique to solve stochastic optimization and stability problems [11], [12]. This technique has also been used to solve energy management problems in [13] and provided online solutions with performance bounds. Motivated by the seminal work, we first adopt the concept of virtual queues as in prior work [13] and introduce two virtual queues: QoSE virtual queues and battery virtual queues to transform the QoSE control problem and battery management problem to queue stability problems. Second, we design an adaptive MG electricity scheduling policy based on the Lyapunov optimization method and prove several deterministic (or "hard") performance bounds for the proposed algorithm. The algorithm can be implemented online because it only relies on the current system status, without needing any future knowledge of the energy demand, supply and price processes. The proposed algorithm also converges exponentially due to the nice property of Lyapunov stability design [14]. The algorithm is evaluated with trace-driven simulations and is shown to achieve significant efficiency on MG operation cost while guaranteeing the residents' QoSE.

The rest of the paper is organized as follows. We present the system model and problem formulation in Section II. An adaptive MG electricity scheduling algorithm is designed and analyzed in Section III. Simulation results are presented and discussed in Section IV. We discuss related work in Section V. Section VI concludes the paper.

\section{System Model and Problem Formulation}

\section{A. System Model}

1) Overview: We consider the electricity supply and consumption in an MG. We assume that the MG is properly designed such that a portion of the electricity demand related to basic living usage (e.g., lighting) from the residents, termed basic usage, can be guaranteed by the minimum capacity of the MG. There are randomness in both electricity supply (e.g., weather change) and demand (e.g., entertainment usage in weekends). To cope with the randomness, the MG works in the grid-connected mode and is equipped with ESSs, such as electrochemical battery, superconducting magnetic energy storage, flywheel energy storage, etc. The ESSs store excess electricity for future use.

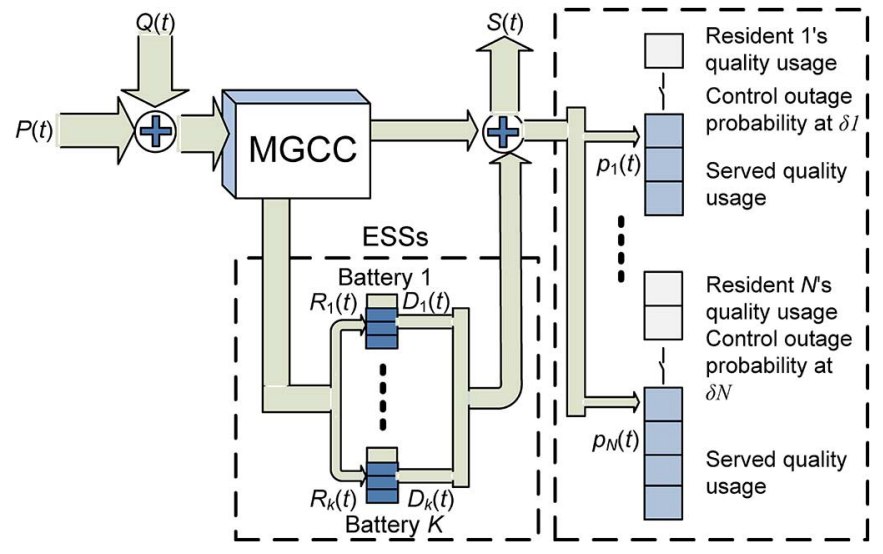

Fig. 1. The system model considered in this paper.

The MGCC collects information about the resident demands, DRER supplies, and ESS levels through the information network. When a resident demand exceeds the pre-agreed level, a quality usage request will be triggered and transmitted to the MGCC. The MGCC will then decide the amount of quality usage to be satisfied with energy from the DRERS, the ESSs, or by purchasing electricity from the macrogrid. The MGCC may also decline some quality usage requests. The excess energy can be stored at the ESSs or sold back to the macrogrid for compensating the cost of MG operation. For brevity, we consider a time-slotted system. The time slot duration is determined by the timescale of the demand and supply processes.

2) Energy Storage System Model: The system model is shown in Fig. 1. Consider a battery farm with $K$ independent battery cells, which can be recharged and discharged. We assume that the batteries are not leaky and do not consider the power loss in recharging and discharging, since the amount is usually small. This assumption can be relaxed by applying a constant percentage of loss to the recharging and discharging processes. For example, if we consider constant charging and discharging loss ratios, we may add a constant percentage on the battery dynamics as $E_{k}(t+1)=\beta \cdot\left(E_{k}(t)-D_{k}(t)+R_{k}(t)\right)$, where $0<\beta \leq 1$. This would not affect the following analysis and algorithm development. For brevity, we also ignore the aging effect of the battery and the maintenance cost, since the cost on the utility market dominates the operation cost of MGs.

Let $E_{k}(t)$ denote the energy level of the $k$ th battery in time slot $t$. The capacity of the battery is bounded as

$$
E_{k}^{\min } \leq E_{k}(t) \leq E_{k}^{\max }, \forall k, t,
$$

where $E_{k}^{\max } \geq 0$ is the maximum capacity, and $E_{k}^{\min } \geq 0$ is the minimum energy level required for battery $k$, which may be set by the battery deep discharge protection settings. The dynamics over time of $E_{k}(t)$ can be described as

$$
E_{k}(t+1)=E_{k}(t)-D_{k}(t)+R_{k}(t), \forall k, t,
$$

where $R_{k}(t)$ and $D_{k}(t)$ are the recharging and discharging energy for battery $k$ in time slot $t$, respectively. The charging and discharging energy in each time slot are bounded as

$$
\begin{cases}0 \leq R_{k}(t) \leq R_{k}^{\max }, & \forall k, t \\ 0 \leq D_{k}(t) \leq D_{k}^{\max }, & \forall k, t .\end{cases}
$$


In each time slot $t, R_{k}(t)$ and $D_{k}(t)$ are determined such that (1) is satisfied in the next time slot.

Usually the recharging and discharging operations cannot be performed simultaneously, which leads to

$$
\left\{\begin{array}{l}
R_{k}(t)>0 \Rightarrow D_{k}(t)=0, \quad \forall k, t \\
D_{k}(t)>0 \Rightarrow R_{k}(t)=0, \quad \forall k, t
\end{array}\right.
$$

3) Energy Supply and Demand Model: Consider $N$ residents in the MG; each generates basic and quality electricity usage requests, and each can tolerate a prescribed outage probability $\delta_{n}$ for the requested quality usage part. The MGCC adaptively serves quality usage requests at different levels to maintain the QoSE as well as the stability of the grid. The service of quality usage can be different for different residents, depending on individual service agreements.

Let $\lambda_{n}$ be the average quality usage arrival rate, and $\delta_{n}$ a prescribed outage tolerance (i.e., a percentage) for user $n$. The average outage rate for the quality usage, $\rho_{n}$, should satisfy

$$
\rho_{n} \leq \delta_{n} \cdot \lambda_{n}
$$

At each time $t$, the quality usage request from resident $n$ is $\alpha_{n}(t) \in\left[0, \alpha_{n}^{\max }\right]$ units, which is an i.i.d random variable with a general distribution and mean $\lambda_{n} \cdot{ }^{1}$ The average rate is $\lambda_{n}=\lim _{t \rightarrow \infty}(1 / t) \sum_{\tau=0}^{t-1} \alpha_{n}(\tau)$. We assume that the users in the MG are rational through the help of smart meters and smart facilities. The average quality usage request rate of the user is specified in the user's contract. The DRERs in the MG generate $U(t)$ units of electricity in time slot $t . U(t)$ can offer enough capacity to support the pre-agreed basic usage in the MG, which is guaranteed by islanded mode MG planning. The electricity is transmitted over power transmission lines. For brevity, we assume the power transmission lines are not subject to outages and the transmission loss is negligible. Let $\alpha_{n}^{b}(t)$ be the pre-agreed basic usage for resident $n$ in time slot $t$, which can be fully satisfied by $U(t)$, i.e., $\sum_{n=1}^{N} \alpha_{n}^{b}(t) \leq U(t)$, for all $t$. In addition, some quality usage request $\alpha_{n}(t)$ may be satisfied if $P(t)=U(t)-\sum_{n=1}^{N} \alpha_{n}^{b}(t) \geq 0$. Let $p_{n}(t)$ be the energy allocated for the quality usage of resident $n$. We have

$$
0 \leq p_{n}(t) \leq \alpha_{n}(t)
$$

We define a function $I_{n}(t) \geq 0$ to indicate the amount of quality usage outage for resident $n$, as $I_{n}(t)=\alpha_{n}(t)-p_{n}(t)$. Then the average outage rate can be evaluated as $\rho_{n}=$ $\lim _{t \rightarrow \infty}(1 / t) \sum_{\tau=0}^{t-1} I_{n}(\tau)$.

The MGCC may purchase additional energy from the macrogrid or sell excess energy back to the macrogrid. Let $Q(t) \in$ $\left[0, Q_{\max }\right]$ denote the energy purchased from the macrogrid and $S(t) \in\left[0, S_{\max }\right]$ the energy sold on the market in time slot $t$, where $Q_{\max }$ and $S_{\max }$ are determined by the capacity of the

\footnotetext{
${ }^{1}$ The Lyapunov optimization method that is used later to solve this problem, is also robust to non-i.i.d. requests (i.e., when a denied demand is requested at a later time). Interested readers can find detailed proofs for the non-i.i.d. case in [15], [16]
}

transformers and power transmission lines. Since it is not reasonable to purchase and sell energy on the market at the same time, we have the following constraints

$$
\left\{\begin{array}{l}
Q(t)>0 \Rightarrow S(t)=0, \quad \forall t \\
S(t)>0 \Rightarrow Q(t)=0, \quad \forall t .
\end{array}\right.
$$

To balance the supply and demand in the MG, we have

$P(t)+Q(t)+\sum_{k=1}^{K} D_{k}(t)-S(t)-\sum_{k=1}^{K} R_{k}(t)=\sum_{n=1}^{N} p_{n}(t), \forall t$.

4) Utility Market Pricing Model: The price for purchasing electricity from the macrogrid in time slot $t$ is $C(t)$ per unit. The purchasing price depends on the utility market state, such as peak/off time of the day. We assume finite $C(t) \in\left[C_{\min }, C_{\max }\right]$, which is announced by the utility market at the beginning of each time slot and remains constant during the slot period [17]. We assume $C(t)$ is independent to the amount of energy to be purchased in that time slot.

If the MGCC determines to sell electric energy on the utility market, the selling price from the market broker is denoted by $W(t) \in\left[W_{\min }, W_{\max }\right]$ in time slot $t$, which is also a stochastic process with a general distribution. We also assume $W(t)$ is known at the beginning of each time slot and independent to the amount of energy to be sold on the market. We assume $C_{\max } \geq$ $W_{\max }, C_{\min } \geq W_{\min }$ and $C(t)>S(t)$ for all $t$. That is, the MG cannot make profit by greedily purchasing energy from the market and then selling it back to the market at a higher price simultaneously.

\section{B. Problem Formulation}

Given the above models, a control policy $A(t)=$ $\left\{Q(t), S(t), R_{k}(t), D_{k}(t), p_{n}(t)\right\}$ is designed to minimize the operation cost of the MG and guarantee the QoSE of the residents. We formulate the electricity scheduling problem as

$$
\begin{aligned}
\text { minimize : } & \lim _{t \rightarrow \infty} \frac{1}{t} \sum_{\tau=0}^{t-1} \mathbb{E}\{Q(\tau) C(\tau)-S(\tau) W(\tau)\} \\
\text { s.t. } & (1)-(8) .
\end{aligned}
$$

As in [13], we assume temporarily that the limit of (9) is well defined by the proposed policy. The lim $\sup _{t \rightarrow \infty}$ operator can be used to capture the limit bound for more general scenarios [15], and the following derivation and results will hold true similarly. Problem (9) is a stochastic programming problem, where the utility prices, generation of DRERs, and consumption of residents are all random. The solution also depends on the evolution of battery states. It is challenging since the supply, demand, and price are all general processes.

1) Virtual Queues: Inspired by prior work [13], we adopt the notation of virtual queues in the problem formulation. We first adopt a battery virtual queue $X_{k}(t)$ that tracks the charge level of each battery $k$ :

$$
X_{k}(t)=E_{k}(t)-D_{k}^{\max }-E_{k}^{\min }-V C_{\max }, \quad \forall k, t,
$$

where $0<V \leq V_{\max }=\min _{k}\left\{\left(E_{k}^{\max }-E_{k}^{\min }-R_{k}^{\max }-\right.\right.$ $\left.\left.D_{k}^{\max }\right) /\left(C_{\max }-W_{\min }\right)\right\}$ is a constant for the trade-off between system performance and ensuring the battery constraints. This 
constant $V_{\max }$ is carefully selected to ensure the evolution of the battery levels always satisfy the battery constraints (1), which will be examined in Section III-C. The virtual queue can be deemed as a shifted version of the battery dynamics in (2) as

$$
X_{k}(t+1)=X_{k}(t)-D_{k}(t)+R_{k}(t), \quad \forall k, t .
$$

These queues are "virtual" because they are maintained by the MGCC control algorithm. Unlike an actual queue, the virtual queue backlog $X_{k}(t)$ may take negative values.

We next introduce a conceptual QoSE virtual queue $Z_{n}(t)$, whose dynamics are governed by the system equation as

$$
Z_{n}(t+1)=\left[Z_{n}(t)-\delta_{n} \cdot \alpha_{n}(t)\right]^{+}+I_{n}(t), \quad \forall n, t .
$$

where $[x]^{+}=\max \{0, x\}$.

Theorem 1: If an MGCC control policy stabilizes the QoSE virtual queue $Z_{n}(t)$, the outage quality usage of resident $n$ will be stabilized at the average QoSE rate $\rho_{n} \leq \delta_{n} \cdot \lambda_{n}$.

Proof: According to the system (12), we have

$$
\left\{\begin{array}{l}
Z_{n}(1) \geq Z_{n}(0)-\delta_{n} \cdot \alpha_{n}(0)+I_{n}(0) \\
\cdots \\
Z_{n}(t) \geq Z_{n}(t-1)-\delta_{n} \cdot \alpha_{n}(t-1)+I_{n}(t-1) .
\end{array}\right.
$$

Summing up the inequalities in (13), we have

$$
Z_{n}(t) \geq Z_{n}(0)-\delta_{n} \cdot \sum_{\tau=0}^{t-1} \alpha_{n}(\tau)+\sum_{\tau=0}^{t-1} I_{n}(\tau) .
$$

Dividing both sides by $t$ and letting $t$ go to infinity, we have

$$
\lim _{t \rightarrow \infty} \frac{Z_{n}(t)-Z_{n}(0)}{t} \geq \lim _{t \rightarrow \infty} \frac{1}{t}\left[-\delta_{n} \sum_{\tau=0}^{t-1} \alpha_{n}(\tau)+\sum_{\tau=0}^{t-1} I_{n}(\tau)\right] .
$$

Note that $Z_{n}(0)$ is finite. If $Z_{n}(t)$ is rate stable by a control policy $I_{n}(t)$, it is finite for all $t$. We have $\lim _{t \rightarrow \infty}\left(\left(Z_{n}(t)-\right.\right.$ $\left.\left.Z_{n}(0)\right) / t\right)=0$, which yields $\rho_{n} \leq \delta_{n} \cdot \lambda_{n}$ due to the definitions of $\lambda_{n}$ and $I_{n}(t)$.

2) Problem Reformulation: With Theorem 1, we can transform the original problem (9) into a queue stability problem with respect to the QoSE virtual queue and the battery virtual queues, which leads to a system stability design from the control theoretic point of view. We have a reformulated stochastic programming problem as follows.

$$
\begin{aligned}
\text { minimize : } & \lim _{t \rightarrow \infty} \frac{1}{t} \sum_{\tau=0}^{t-1} \mathbb{E}\{Q(\tau) C(\tau)-S(\tau) W(\tau)\} \\
\text { s.t. } & (3),(4),(6),(7),(8)
\end{aligned}
$$

Battery and QoSE virtual queue stability

$$
\text { constraints. }
$$

Theorem 1 indicates that QoSE provisioning is equivalent to stabilizing the QoSE virtual queue $Z_{n}(t)$, while stabilizing the virtual queues (11) ensures that the battery constraints (1) are satisfied. We then apply Lyapunov optimization to develop an adaptive electricity scheduling policy for problem (15), in which the policy greedily minimizes the Lyapunov drift in every time slot $t$ to push the system toward stability. Note that following the reformulation and Lyapunov optimization framework in [13],
[29], the solution of the reformulated problem (15) approximately solves the original problem (9). But we will show that the solution derived in the following has bounded optimality as given in Theorem 4.

\section{Lyapunov Optimization}

We define the Lyapunov function for system state $\vec{\Theta}(t)=$ $[\vec{X}(t), \vec{Z}(t)]^{T}$ with dimension $(N+K) \times 1$ as follows, in which $\vec{X}(t)=\left[X_{1}(t) \cdots X_{K}(t)\right]^{T}$ and $\vec{Z}(t)=\left[Z_{1}(t) \cdots Z_{N}(t)\right]^{T}$.

$$
L(\vec{\Theta}(t))=\frac{1}{2} \sum_{k=1}^{K}\left[X_{k}(t)\right]^{2}+\frac{1}{2} \sum_{n=1}^{N}\left[Z_{n}(t)\right]^{2},
$$

which is positive definite, since $L(\vec{\Theta}(t))>0$ when $\vec{\Theta}(t) \neq \overrightarrow{\mathbf{0}}$ and $L(\vec{\Theta}(t))=0 \Leftrightarrow \vec{\Theta}(t)=\overrightarrow{\mathbf{0}}$. We then define the conditional one slot Lyapunov drift as

$$
\Delta(\vec{\Theta}(t))=\mathbb{E}\{L(\vec{\Theta}(t+1))-L(\vec{\Theta}(t)) \mid \vec{\Theta}(t)\} .
$$

With the drift defined as in (17), it can be shown that

$$
\begin{aligned}
\Delta(\vec{\Theta}(t))= & \frac{1}{2} \mathbb{E}\left\{\sum_{k=1}^{K}\left[\left(X_{k}(t+1)\right)^{2}-\left(X_{k}(t)\right)^{2} \mid X_{k}(t)\right]\right. \\
& \left.+\sum_{n=1}^{N}\left[\left(Z_{n}(t+1)\right)^{2}-\left(Z_{n}(t)\right)^{2} \mid Z_{n}(t)\right]\right\} \\
\leq & B+\sum_{n=1}^{N} \mathbb{E}\left\{Z_{n}(t)\left(1-\delta_{n}\right) \alpha_{n}(t) \mid Z_{n}(t)\right\} \\
& +\sum_{k=1}^{K} \mathbb{E}\left\{X_{k}(t)\left(R_{k}(t)-D_{k}(t)\right) \mid X_{k}(t)\right\} \\
& -\sum_{n=1}^{N} \mathbb{E}\left\{\left(Z_{n}(t)+\alpha_{n}(t)\right) p_{n}(t) \mid Z_{n}(t)\right\}
\end{aligned}
$$

where

$B=(1 / 2) \sum_{k=1}^{K}\left(\max \left\{D_{k}^{\max }, R_{k}^{\max }\right\}\right)^{2}+(1 / 2) \sum_{n=1}^{N}(2+$ $\left.\delta_{n}^{2}\right)\left(\alpha_{n}^{\max }\right)^{2}$ is a constant. $X_{k}(t+1)$ and $Z_{n}(t+1)$ are replaced by the virtual queue dynamics (11) and (12). Since $X_{k}(t)$ and $Z_{n}(t)$ are known at time slot $t$, they can be explicitly taken out of the expectation operation. The derivation of (18) is given in Appendix A.

To minimize the operation cost of the MG, we adopt the drift-plus-penalty method [12]. Specifically, we select the control policy $\mathrm{A}(t)=\left\{Q(t), S(t), R_{k}(t), D_{k}(t), p_{n}(t)\right\}$ to minimize the bound on the drift-plus-penalty as:

$$
\begin{aligned}
& \Delta(\vec{\Theta}(t))+V \mathbb{E}\{Q(t) C(t)-S(t) W(t) \mid \vec{\Theta}(t)\} \\
\leq & \text { right - hand - side of }(18) \\
& +V \mathbb{E}\{Q(t) C(t)-S(t) W(t) \mid \vec{\Theta}(t)\},
\end{aligned}
$$

where $0<V \leq V_{\max }$ is defined in Section II-B1 for the trade-off between stability performance and operation cost minimization. In (19), the new objective function jointly considers battery and QoSE stability (the first term) and MG cost minimization (the second term) under the condition of current system states. The parameter $V$ is a nonnegative constant to provide the performance tradeoff of the two terms. If $V=0$, it corresponds to the pure system stability problem 
by minimization the Lyapunov drift (17). Generally, we select $0<V \leq V_{\max }$ for weighting the cost minimization term in control decision, which provides a tradeoff between cost minimization and stability.

Given the current virtual queue states $X_{k}(t)$ and $Z_{n}(t)$, market prices $S(t)$ and $W(t)$, available DRERs energy $P(t)$, and the resident quality usage request $\alpha_{n}(t)$, the optimal policy is the solution to the following problem.

$$
\begin{aligned}
\text { minimize : } & B+\sum_{n=1}^{N}\left[Z_{n}(t)\left(1-\delta_{n}\right) \alpha_{n}(t)\right] \\
& +V[Q(t) C(t)-S(t) W(t)] \\
& +\sum_{k=1}^{K}\left[X_{k}(t)\left(R_{k}(t)-D_{k}(t)\right)\right] \\
& -\sum_{n=1}^{N}\left[\left(Z_{n}(t)+\alpha_{n}(t)\right) p_{n}(t)\right] \\
\text { s.t. } & (3),(4),(6),(7),(8) .
\end{aligned}
$$

Since the control policy $A(t)$ is only applied to the last three terms of (20), we can further simplify problem (20) as

$$
\begin{aligned}
\operatorname{minimize}: \quad & V[Q(t) C(t)-S(t) W(t)] \\
& +\sum_{k=1}^{K}\left[X_{k}(t)\left(R_{k}(t)-D_{k}(t)\right)\right] \\
& -\sum_{n=1}^{N}\left[\left(Z_{n}(t)+\alpha_{n}(t)\right) p_{n}(t)\right] \\
\text { s.t. } & (3),(4),(6),(7),(8),
\end{aligned}
$$

which is a mixed-integer optimization problem and can be solved based on observations of the current system state $\left\{X_{k}(t), Z_{n}(t), C(t), W(t), P(t), \alpha_{n}(t)\right\}$.

\section{ELECTRICITY SCHEDULING ALGORITHM}

\section{A. Properties of Optimal Scheduling}

With the Lyapunov penalty-and-drift method, we transform problem (15) to problem (21) to be solved for each time slot. We have the following properties for the optimal scheduling. The proofs of the Lemmas are provided in Appendices B-D.

Lemma 1: The optimal solution to problem (21) has the following properties:

1) If $Q(t)>0$, we have $S(t)=0$,

a) If $X_{k}(t)>-V C(t)$, the optimal solution always selects $R_{k}(t)=0$; if $X_{k}(t)<-V C(t)$, the optimal solution always selects $D_{k}(t)=0$.

b) If $Z_{n}(t)>V C(t)-\alpha_{n}(t)$, the optimal solution always selects $p_{n}(t) \geq\left(1-\delta_{n}\right) \alpha_{n}(t)$; if $Z_{n}(t)<$ $V C(t)-\alpha_{n}(t)$, the optimal solution always selects $p_{n}(t)=0$.

2) When $Q(t)=0$, we have $S(t)>0$,

a) If $X_{k}(t)>-V W(t)$, the optimal solution always selects $R_{k}(t)=0$; if $X_{k}(t)<-V W(t)$, the optimal solution always selects $D_{k}(t)=0$.

b) If $Z_{n}(t)>V W(t)-\alpha_{n}(t)$, the optimal solution always selects $p_{n}(t) \geq\left(1-\delta_{n}\right) \alpha_{n}(t)$; if $Z_{n}(t)<$
$V W(t)-\alpha_{n}(t)$, the optimal solution always selects $p_{n}(t)=0$.

Lemma 2: The optimal solution to the battery management problem has the following properties:

1) If $X_{k}(t)>-V W_{m i n}$, the optimal solution always selects $R_{k}(t)=0$.

2) If $X_{k}(t)<-V C_{\max }$, the optimal solution always selects $D_{k}(t)=0$.

Lemma 3: The optimal solution to the QoSE provisioning problem has the following properties:

1) If $Z_{n}(t)>V C_{\max }$, the optimal solution always selects $p_{n}(t) \geq\left(1-\delta_{n}\right) \alpha_{n}(t)$.

2) If $Z_{n}(t)<V W_{\min }-\alpha_{n}^{\max }$, the optimal solution always selects $p_{n}(t)=0$.

Lemma 1 provides useful insights for simplifying the algorithm design, which will be discussed in Section III-B. The intuition behind these lemmas is two-fold. On the ESS management side, if either the purchasing price $C(t)$ or the selling price $W(t)$ is low, the MG prefers to recharge the ESSs to store excess electricity for future use. On the other hand, if either $C(t)$ or $W(t)$ is high, the MG is more likely to discharge the ESSs to reduce the amount of energy to purchase or sell more stored energy back to the macrogrid. On the QoSE provisioning side, if either $C(t)$ or $W(t)$ is high and the quality usage $\alpha_{n}(t)$ is low, the MG is apt to decline the quality usage for lower operation cost. On the other hand, if either $C(t)$ or $W(t)$ is low and $\alpha_{n}(t)$ is high, the quality usage are more likely to be granted by purchasing more energy or limiting the sell of energy.

\section{B. MG Optimal Scheduling Algorithm}

In this section, we present the MG control policy $A(t)$ to solve problem (21). Given the current virtual queue state $\left\{X_{k}(t), Z_{n}(t)\right\}$, market prices $C(t)$ and $W(t)$, quality usage $\alpha_{n}(t)$ and available energy $P(t)$ from the DRERS for serving quality usage, problem (21) can be decomposed into the following two linear programming (LP) sub-problems [since one of $S(t)$ and $Q(t)$ must be zero, see (7)].

$$
\begin{aligned}
\text { minimize : } & V Q(t) C(t)+\sum_{k=1}^{K}\left[X_{k}(t)\left(R_{k}(t)-D_{k}(t)\right)\right] \\
& -\sum_{n=1}^{N}\left(\left(Z_{n}(t)+\alpha_{n}(t)\right) p_{n}(t)\right) \\
\text { s.t. } \quad & S(t)=0,(3),(4),(6),(8) . \\
\text { minimize : } \quad & -V S(t) W(t)+\sum_{k=1}^{K}\left[X_{k}(t)\left(R_{k}(t)-D_{k}(t)\right)\right] \\
& -\sum_{n=1}^{N}\left(\left(Z_{n}(t)+\alpha_{n}(t)\right) p_{n}(t)\right) \\
\text { s.t. } & Q(t)=0,(3),(4),(6),(8) .
\end{aligned}
$$

In sub-problem (22), we set $R_{k}(t)=0$ if $X_{k}(t)>-V C(t)$, and $D_{k}(t)=0$ if $X_{k}(t)<-V C(t)$ according to Lemma 1. Also, if $Z_{n}(t)<V C(t)-\alpha_{n}(t)$, we set $p_{n}(t)=0$; otherwise, we reset constraint (6) to a smaller search space of (1$\left.\delta_{n}\right) \alpha_{n}(t) \leq p_{n}(t) \leq \alpha_{n}(t)$. We take a similar approach for solving sub-problem (23) by replacing $C(t)$ with $W(t)$. Then 
we compare the objective values of the two sub-problems and select the more competitive solution as the MG control policy. The complete algorithm is presented in Algorithm 1.

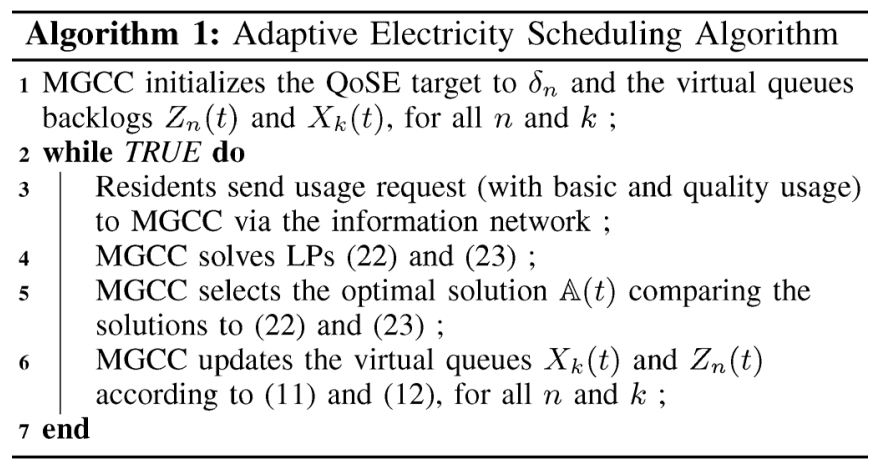

\section{Performance Analysis}

The proposed scheduling algorithm dynamically balances cost minimization and QoSE provisioning. It only requires current system state information (i.e., as an online algorithm). The algorithm is also robust to non-i.i.d. and non-ergodic behaviors of the processes (see, e.g., [18], [13]).

Theorem 2: The constraint on the ESS battery level $E_{k}(t)$, $E_{k}^{\text {min }} \leq E_{k}(t) \leq E_{k}^{\max }$, is always satisfied for all $k$ and $t$.

Proof: From the battery virtual queue definition (10), the constraint $E_{k}^{\min } \leq E_{k}(t) \leq E_{k}^{\max }$ is equivalent to

$-V C_{\max }-D_{k}^{\max } \leq X_{k}(t) \leq E_{k}^{\max }-V C_{\max }-D_{k}^{\max }-E_{k}^{\min }$.

We assume all the batteries satisfy the battery capacity constraint at the initial time $t=0$, i.e., $E_{k}^{\min } \leq E_{k}(0) \leq E_{k}^{\max }$, for all $k$. Supposing the inequalities hold true for time $t$, we then show the inequalities still hold true for time $t+1$.

First, we show $X_{k}(t+1) \leq E_{k}^{\max }-V C_{\max }-D_{k}^{\max }-E_{k}^{\min }$. If $-V W_{\min }<X_{k}(t) \leq E_{k}^{\max }-V C_{\max }-D_{k}^{\max }-E_{k}^{\min }$, then with $X_{k}(t)>-V W_{\min } \Rightarrow R_{k}(t)=0$ from Lemma 2, we have $X_{k}(t+1)=X_{k}(t)-D_{k}(t) \leq X_{k}(t) \leq E_{k}^{\max }-V C_{\max }-$ $D_{k}^{\text {max }}-E_{k}^{\text {min }}$. If $X_{k}(t) \leq-V W_{\min }$, then the largest value is $X_{k}(t+1)=-V W_{\min }+R_{k}^{\max }$. For any $0<V \leq V_{\max }$, we have

$$
\begin{aligned}
& E_{k}^{\text {max }}-V C_{\max }-D_{k}^{\text {max }}-E_{k}^{\text {min }} \\
\geq & E_{k}^{\text {max }}-\min _{k}\left\{\frac{E_{k}^{\max }-E_{k}^{\text {min }}-R_{k}^{\text {max }}-D_{k}^{\max }}{C_{\max }-W_{\min }}\right\} C_{\max } \\
& -D_{k}^{\max }-E_{k}^{\text {min }} \geq R_{k}^{\max } \geq X_{k}(t+1) .
\end{aligned}
$$

It follows that $X_{k}(t+1) \leq E_{k}^{\max }-V C_{\max }-D_{k}^{\max }-E_{k}^{\min }$.

Next, we show $X_{k}(t+1) \geq-V C_{\max }-D_{k}^{\max }$. Assuming $-V C_{\max }-D_{k}^{\max } \leq X_{k}(t) \leq-V C_{\max }$, then from Lemma 2 , we have $X_{k}(t) \leq-V C_{\max } \Rightarrow D_{k}(t)=0$. It follows that

$$
X_{k}(t+1)=X_{k}(t)+R_{k}(t) \geq X_{k}(t) \geq-V C_{\max }-D_{k}^{\max }
$$

If $X_{k}(t) \geq-V C_{\max }$, following (10), we have

$$
\begin{aligned}
X_{k}(t+1) & =X_{k}(t)-D_{k}(t)+R_{k}(t) \geq X_{k}(t)-D_{k}^{\max } \\
& \geq-V C_{\text {max }}-D_{k}^{\max } .
\end{aligned}
$$

Therefore, we have $X_{k}(t+1) \geq-V C_{\max }-D_{k}^{\max }$. Thus the inequalities also hold true for time $t+1$.
It follows that $E_{k}^{\text {min }} \leq E_{k}(t) \leq E_{k}^{\max }$ is satisfied under the optimal scheduling algorithm for all $k, t$.

Theorem 3: The worst-case backlogs of the QoSE virtual queue for each resident $n$ is bounded by $Z_{n}(t) \leq Z_{n}^{\max }=$ $V C_{\max }+\alpha_{n}^{\max }$, for all $n, t$. Moreover, the worst-case average amount of outage of quality usage for resident $n$ in a period $T$ is upper bounded by $Z_{n}^{\max }+T \delta_{n} \alpha_{n}^{\max }$.

Proof: i) We first prove the upper bound $Z_{n}^{\max }$. Initially, we have $Z_{n}(0)=0 \leq V C_{\max }+\alpha_{n}^{\max }$. Assume that in time slot $t$ the backlog of the QoSE virtual queue of resident $n$ satisfies $Z_{n}(t) \leq Z_{n}^{\max }=V C_{\max }+\alpha_{n}^{\max }$. We then check the backlog at time $t+1$ and show the bound still holds true.

If $Z_{n}(t)>V C_{\max }$, following Lemma 3 , the optimal scheduling for the quality usage of resident $n$ satisfies $p_{n}(t) \geq(1-$ $\left.\delta_{n}\right) \alpha_{n}(t)$. From the virtual queue dynamics (12), we have

$$
Z_{n}(t+1) \leq\left[Z_{n}(t)-\delta_{n} \alpha_{n}(t)\right]^{+}+\delta_{n} \alpha_{n}(t) .
$$

If $Z_{n}(t) \geq \delta_{n} \alpha_{n}(t)$, we have $Z_{n}(t+1) \leq Z_{n}(t) \leq V C_{\max }+$ $\alpha_{n}^{\max }$; otherwise, it follows that $Z_{n}(t+1) \leq \delta_{n} \alpha_{n}(t)<$ $V C_{\max }+\alpha_{n}^{\max }$.

If $Z_{n}(t) \leq V C_{\max }$, we have $Z_{n}(t+1) \leq\left[Z_{n}(t)-\right.$ $\left.\delta_{n} \alpha_{n}(t)\right]^{+}+\alpha_{n}^{\max }$. If $Z_{n}(t) \geq \delta_{n} \alpha_{n}(t)$, we have $Z_{n}(t+1) \leq Z_{n}(t)-\delta_{n} \alpha_{n}(t)+\alpha_{n}^{\max } \leq V C_{\max }+\alpha_{n}^{\max } ;$ otherwise, we have $Z_{n}(t+1) \leq \alpha_{n}^{\max } \leq V C_{\max }+\alpha_{n}^{\max }$.

Thus we have $Z_{n}(t+1) \leq Z_{n}^{\max }=V C_{\max }+\alpha_{n}^{\max }$. The proof of the QoSE virtual queue backlog bound is completed.

ii) Consider an interval $\left[t_{1}, t_{2}\right]$ with length of $T$. Summing (12) from $t_{1}$ to $t_{2}$, we have $Z_{n}\left(t_{2}+1\right) \geq Z_{n}\left(t_{1}\right)-$ $\delta_{n} \sum_{\tau=t_{1}}^{t_{2}} \alpha_{n}(\tau)+\sum_{\tau=t_{1}}^{t_{2}}\left[\alpha_{n}(\tau)-p_{n}(\tau)\right] \geq \sum_{\tau=t_{1}}^{t_{2}}\left[\alpha_{n}(\tau)-\right.$ $\left.p_{n}(\tau)\right]-T \delta_{n} \alpha_{n}^{\max }$. It follows that

$$
\sum_{\tau=t_{1}}^{t_{2}}\left[\alpha_{n}(\tau)-p_{n}(\tau)\right] \leq Z_{n}^{\max }+T \delta_{n} \alpha_{n}^{\max } .
$$

Theorem 4: The average MG operation cost under the adaptive electricity scheduling algorithm in Algorithm $1, y$, is bounded as $y \leq y^{*}+B^{*} / V$, where $y^{*}$ is the optimal operating cost of the original problem (9) and $B^{*}=B+\sum_{n=1}^{N} Z_{n}^{\max }\left(1-\delta_{n}\right) \alpha_{n}^{\max }$.

Proof: From Theorem 2, the battery capacity constraints is met in each time slot with the adaptive control policy. Take expectation on (2) and sum it over the period $[0, t-1]$ :

$\mathbb{E}\left\{E_{k}(t)\right\}-\mathbb{E}\left\{E_{k}(0)\right\}=\sum_{\tau=0}^{t-1}\left[\mathbb{E}\left\{R_{k}(\tau)\right\}-\mathbb{E}\left\{D_{k}(\tau)\right\}\right], \forall k$.

Since $E_{k}^{\min } \leq E_{k}(t) \leq E_{k}^{\max }$, we divide both sides by $t$ and let $t$ go to infinity, to obtain

$$
\lim _{t \rightarrow \infty} \frac{1}{t} \sum_{\tau=0}^{t-1} \mathbb{E}\left\{R_{k}(\tau)\right\}=\lim _{t \rightarrow \infty} \frac{1}{t} \sum_{\tau=0}^{t-1} \mathbb{E}\left\{D_{k}(\tau)\right\}, \quad \forall k .
$$

Consider the following relaxed version of problem (9).

$$
\begin{aligned}
\operatorname{minimize}: & \lim _{t \rightarrow \infty} \frac{1}{t} \sum_{\tau=0}^{t-1} \mathbb{E}\{Q(\tau) C(\tau)-S(\tau) W(\tau)\} \\
\text { s.t. } & (3),(4),(5),(6),(7),(8), \text { and }(24) .
\end{aligned}
$$


Since the constraints in problem (25) are relaxed from that in problem (9), the optimal solution to problem (9) is also feasible for problem (25). The solution of (25) does not depend on battery energy levels. Let the optimal solution for problem (25) be $\hat{A}(t)=\left\{\hat{Q}(t), \hat{S}(t), \hat{R}_{k}(t), \hat{D}_{k}(t), \hat{p}_{n}(t)\right\}$ and the corresponding object value is $\hat{y} \leq y^{*}$. According to the properties of optimality of stationary and randomized policies [19], the optimal solution $\hat{\mathrm{A}}(t)$ satisfies $\mathbb{E}\left\{\hat{R}_{k}(t)-\hat{D}_{k}(t)\right\}=0$ and $\hat{y}=\mathbb{E}\{\hat{Q}(\tau) C(\tau)-\hat{S}(\tau) W(\tau)\}$.

We substitute solution $\hat{A}(t)$ into the right-hand-side of the drift-and-penalty (19). Since our proposed policy minimizes the right-hand-side of (19), we have

$$
\begin{gathered}
\Delta(\vec{\Theta}(t))+V \mathbb{E}\{Q(t) C(t)-S(t) W(t) \mid \vec{\Theta}(t)\} \\
\leq B+\sum_{n=1}^{N} \mathbb{E}\left\{Z_{n}(t)\left(1-\delta_{n}\right) \alpha_{n}(t) \mid Z_{n}(t)\right\} \\
\quad+\sum_{k=1}^{K} X_{k}(t) \mathbb{E}\left\{\hat{R}_{k}(t)-\hat{D}_{k}(t) \mid X_{k}(t)\right\} \\
\quad-\sum_{n=1}^{N}\left(Z_{n}(t)+\alpha_{n}(t)\right) \mathbb{E}\left\{\hat{p}_{n}(t) \mid Z_{n}(t)\right\} \\
\quad+V \mathbb{E}\{\hat{Q}(t) C(t)-\hat{S}(t) W(t) \mid \vec{\Theta}(t)\} \\
\leq B+\sum_{n=1}^{N} Z_{n}^{\max }\left(1-\delta_{n}\right) \alpha_{n}^{\text {max }}+V \cdot y^{*}
\end{gathered}
$$

The second inequality is due to the stationary and randomized policy $\hat{A}[19], \mathbb{E}\left\{\hat{R}_{k}(t)-\hat{D}_{k}(t)\right\}=0,0 \leq Z_{n}(t) \leq Z_{n}^{\max }$, $\alpha_{n}(t) \geq 0, p_{n}(t) \geq 0$, and $\hat{y} \leq y^{*}$. Taking expectation and sum up from 0 to $T-1$, we obtain

$$
\begin{aligned}
& \sum_{t=0}^{T-1} V \mathbb{E}\{Q(t) C(t)-S(t) W(t)\} \\
\leq & T \cdot B^{*}+T \cdot V \cdot y^{*}-\mathbb{E}\{L(\vec{\Theta}(T))\}+\mathbb{E}\{L(\vec{\Theta}(0))\} \\
\leq & T \cdot B^{*}+T \cdot V \cdot y^{*}+\mathbb{E}\{L(\vec{\Theta}(0))\} .
\end{aligned}
$$

The second inequality is due to the nonnegative property of Lyapunove functions. Divide both sides by $V \cdot T$ and let $T$ go to infinity. Since the initial system state $\vec{\Theta}(0)$ is finite, we have $\lim _{T \rightarrow \infty}(1 / T) \sum_{t=0}^{T-1} V \mathbb{E}\{Q(t) C(t)-S(t) W(t)\} \leq y^{*}+$ $\left(B^{*} / V\right)$.

It is worth noting that the choice of $V$ controls the optimality of the proposed algorithm. Specifically, a larger $V$ leads to a tighter optimality gap. However, from the proof of Theorem 2, $V$ is limited by $V_{\max }$, which ensures the feasibility of the battery constraints. This is actually a similar phenomenon to the so-called performance-congestion trade-off [19]. Through the definition of $V_{\max }$ (see Section II-B1), it can be seen that if we invest more on the individual storage components for a larger ESS capacity, the proposed algorithm can achieve a better performance (i.e., a smaller optimality gap).

The proposed algorithm solves the relaxed drift-plus-penalty problem (21), and its solution is suboptimal. However, we proved that the optimality gap of the solution is bounded by
$B^{*} / V$. It is also worth noting that all the performance bounds of the proposed algorithm are deterministic, which provide "hard" guarantees for the performance of the proposed adaptive scheduling policy in every time slot. Unlike probabilistic approaches, the proposed method provides useful guidelines for the MG design, while guaranteeing the MG operation cost, grid stability, and the usage quality of residents.

\section{Simulation Study}

We demonstrate the performance of the proposed adaptive MG electricity scheduling algorithm through extensive simulations. We simulated an MG with 500 residents, where the electricity from DRERs is supplied by a wind turbine plant. We use the renewable energy supply data from the Western Wind Resources Dataset published by the National Renewable Energy Laboratory [20]. The ESSs consists of 100 PHEV Li-ion battery packs, each of which has a maximum capacity of $16 \mathrm{kWh}$ and the minimum energy level is 0 . The battery can be fully charged or discharged within 2 hours [21].

The residents' pre-agreed power demand is uniformly distributed in $[2 \mathrm{~kW}, 25 \mathrm{~kW}]$, and the quality usage power is uniformly distributed in $[0,10 \mathrm{~kW}$. The MG works in the gridconnected mode and may purchase/sell electricity from/to the macrogrid. The utility prices in the macrogrid are obtained from [22] and are time-varying. We assume the selling price by the broker is uniformly random and below the purchasing price in each time slot. The time slot duration is 15 minutes. The MGCC serves a certain level of quality usage according to the adaptive electricity scheduling policy. The QoSE target is set to $\delta_{n}=0.07$ for all residents. The control parameter is $V=V_{\max }$, unless otherwise specified.

\section{A. Algorithm Performance}

We first investigate the average QoSEs and total MG operation cost with default settings for a five-day period. We use the MATLAB LP solver for solving the sub-problems (22) and (23). For better illustration, we only show the QoSEs of three randomly chosen users in Fig. 2. It can be seen that all the average QoSEs converge to the neighborhood of 0.08 within 200 time slots, which is close to the MG requested criteria $\delta_{n}=0.07$. In fact the proposed scheme converges exponentially, due to the inherent exponential convergence property in Lyapunov stability based design [14].

We also plot the MG operation traces from this simulation in Fig. 5. The energy for serving quality usage from the DEREs are plotted in Fig. 5(a). It can be seen that the DRERs generate excessive electricity from slot 150 to 200 , which is more than enough for the residents. Thus, the MGCC sells more electricity back to the macrogrid and obtains significant cost compensation accordingly. In Fig. 5(b), we plot the traces of electricity trading, where the positive values are the purchased electricity (marked as brown bars), and the negative values represent the sold electricity (marked as dark blue bars). The MG operation costs are plotted in Fig. 5(c). The curve rises when the MG purchases electricity and falls when the MG sells electricity. From slot 150 to 200 , the operation cost drops significantly due to 


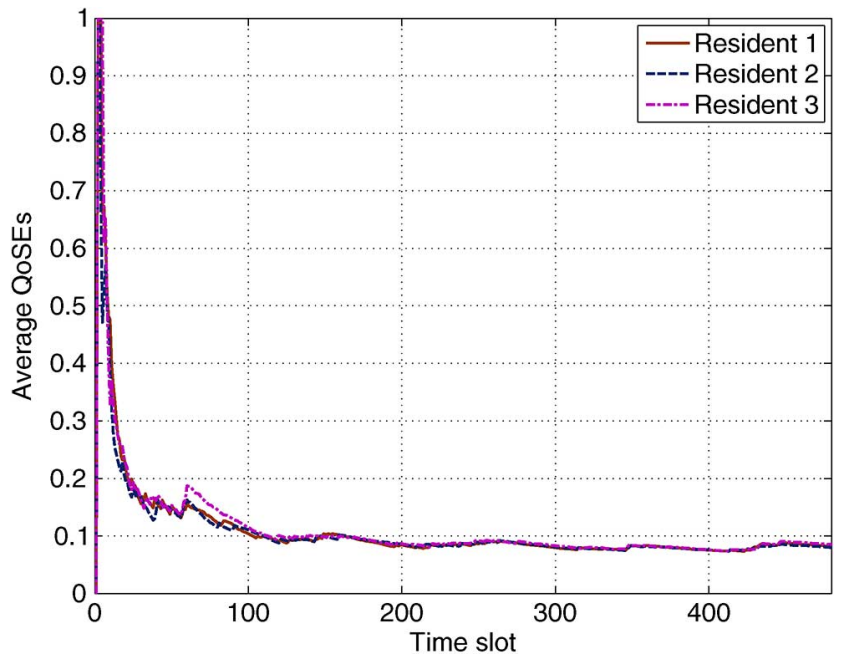

Fig. 2. Average QoSEs for resident 1,2 , and $3\left(V=V_{\max }\right)$.

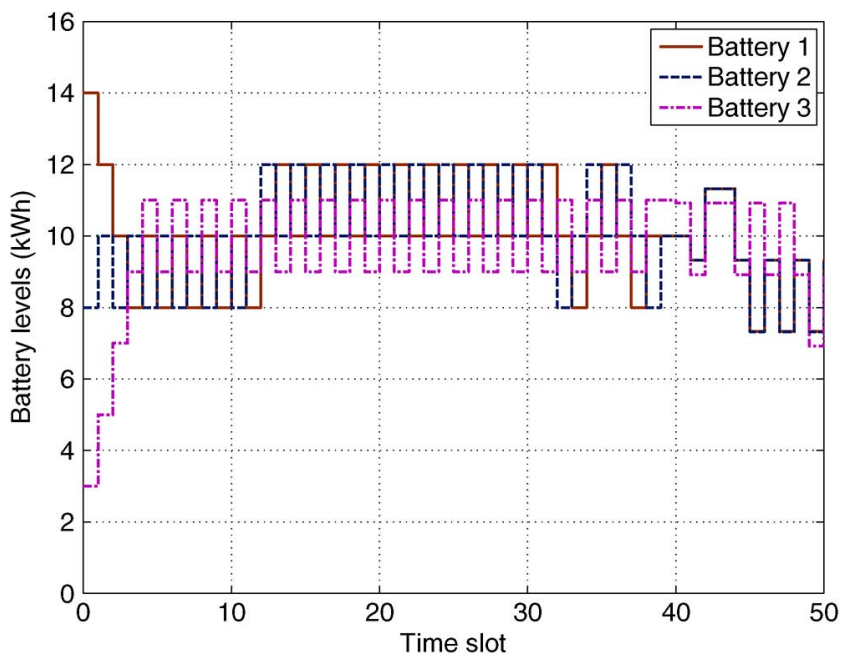

Fig. 3. Energy levels of three Li-ion batteries $\left(V=V_{\max }\right)$.

profits of selling excess electricity from the DEREs. The operation cost is $\$ 418.10$ by the end of the period, which means the net spending of the MG is $\$ 418.10$ on the utility market.

We then examine the energy levels of the batteries in Fig. 3. We only plot the levels of three batteries in the first 50 time slots for clarity. The proposed control policy charges and discharges the batteries in the range of 0 to $16 \mathrm{kWh}$, which falls strictly within the battery capacity limit. It can be seen that the amount of energy for charging or discharging in one slot is limited by $2 \mathrm{kWh}$ in the figure, due to the short time slots comparing to the 2-hour fully charge/discharge periods. For longer time slot durations and batteries with faster charge/discharge speeds, the variation of the energy level in Fig. 3 could be higher. However, Theorem 2 indicates that the feasibility of the battery management constraint is always ensured, if the control parameter $V$ satisfies $0<V \leq V_{\max }$.

We next evaluate the performance of the proposed adaptive control algorithm under different values of control parameter $V$. For different values $V=\left\{V_{\max }, V_{\max } / 2, V_{\max } / 4\right\}$, the QoSEs are stabilized at $0.081,0.061$, and 0.055 , and the total

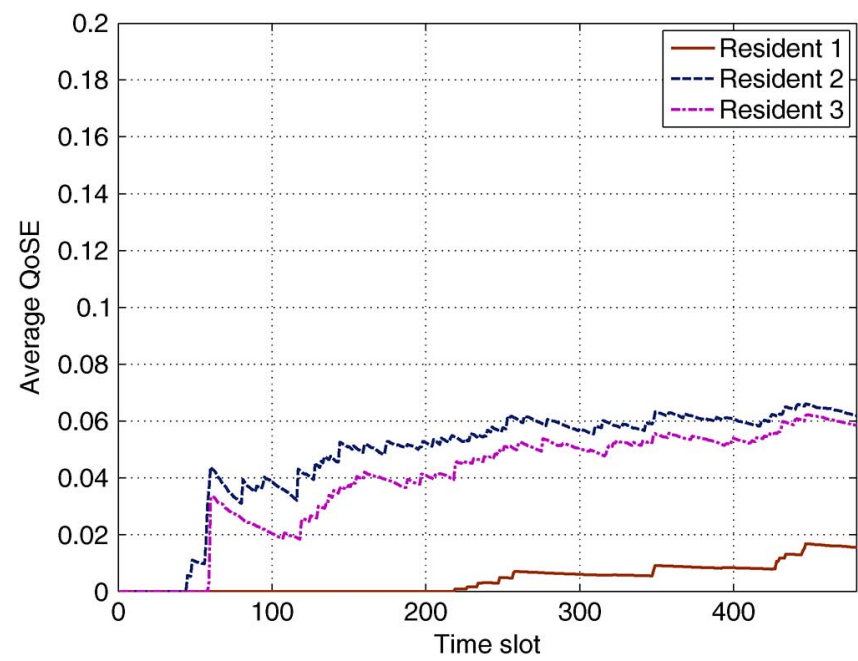

Fig. 4. QoSEs for three residents with different service contracts $(V=$ $\left.V_{\max } / 2\right)$.

operation cost are $\$ 418.10, \$ 625.69$, and $\$ 717.75$, respectively. We find the QoSE decreases from 0.081 to 0.055 , while the total operation cost is increased from $\$ 418.10$ to $\$ 717.75$, as $V_{\max }$ is decreased. This demonstrates the performance-congestion trade-off as in Theorem 4: a larger $V$ leads to a smaller objective value (i.e., the operating cost), but the system is also penalized by a larger virtual queue backlog, which corresponds to a higher QoSE. On the contrary, a smaller $V$ favors the resident quality usage, but increases the total operation cost. In practice, we can select a proper value for this parameter based on the MG design specifications.

It would be interesting to examine the case where the residents require different QoSEs. We assume five residents with a service contract for lower QoSEs. We plot the average QoSEs of three residents with $V=V_{\max } / 2$ in Fig. 4. Resident 1 prefers an outage probability $\delta_{1}=0.02$, while residents 2 and 3 require an outage probability $\delta_{2}=\delta_{3}=0.07$. It can be seen in Fig. 4 that resident 1's QoSE converges to 0.015 , while the other two residents' QoSEs remains around 0.063 .

\section{B. Comparison With a Benchmark}

We compare the performance of the proposed scheme with a heuristic MG electricity control policy (MECP) (as a benchmark). In MECP, the MGCC blocks quality usage requests simply by tossing a coin with the target probability. We use $\delta_{n}=0.03$ in the simulations. If there is sufficient electricity from the DRERs, all the quality usage requests will be granted and the excess energy will be stored in the ESSs. If there is still any surplus energy, the MGCC will sell it to macrogrid. If there is insufficient electricity from the DRERs, the ESSs will be discharged to serve the quality usage requests. The MGCC will purchase electricity from macrogrid if even more electricity is required. Finally, with a predefined probability, e.g., 0.5 in the following simulation, the MG purchases as much energy as possible to charge the ESSs.

We run 100 simulations with different random seeds for a seven-day period. We assume in the first five days the resident behavior is the same as previous default settings. In the last two 


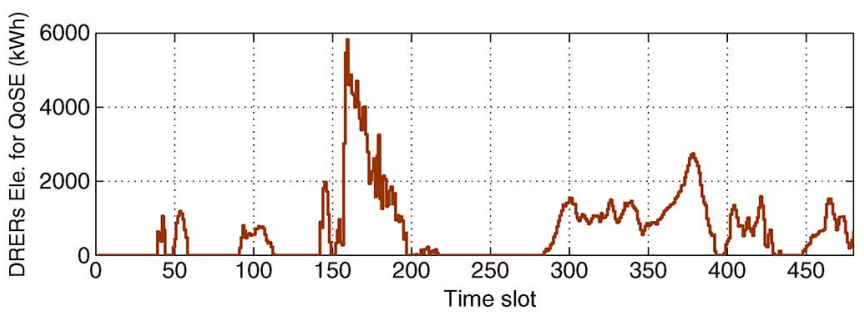

(A)

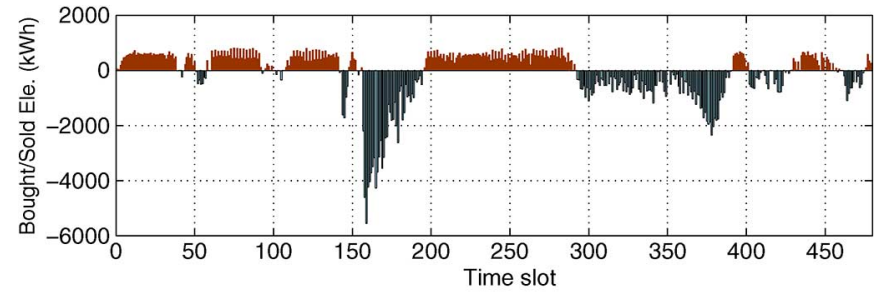

(B)

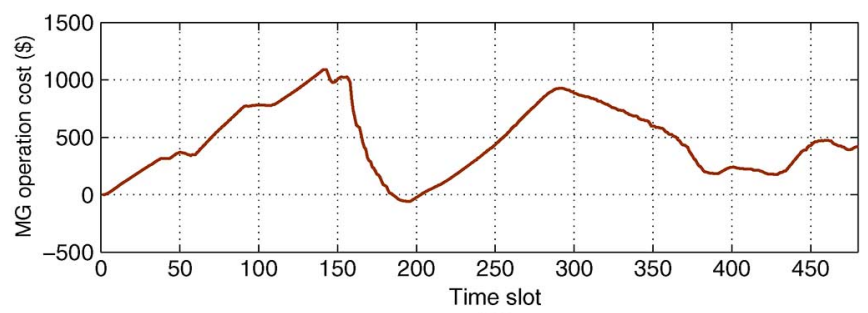

(C)

Fig. 5. MG operation traces of the proposed algorithm for the 5-day period.

days, we assume the residents are apt to request more electricity (e.g., more activities in weekends). In the last two days the resident pre-agreed basic usage power demand is uniformly distributed from $5 \mathrm{~kW}$ to $35 \mathrm{~kW}$. The quality usage power is uniformly distributed from 0 to $20 \mathrm{~kW}$.

We find that the proposed algorithm earns $\$ 947.27$ from the utility market (with $95 \%$ confidence interval $[950.65,943.89]$ ). The profit mainly comes from the abundant DRER generation in the last two days. MECP only earns $\$ 379.74$ from the market (with $95 \%$ confidence interval $[387.96,371.52]$ ), which is $60 \%$ lower than that of the proposed control policy. We also find that the QoSEs under the proposed control policy remains about 0.025 , which is lower than the criteria $\delta_{n}=0.03$. This is because there are a sudden price jump from $\$ 27 / \mathrm{MWh}$ to $\$ 356 / \mathrm{MWh}$ in the afternoon of the last day. This sharp increment increases $C_{\max }$ eight times and decreases the value of $V_{\max }$. Due to the performance-congestion trade-off, the QoSEs become smaller (lower than MECP's 0.03 level).

\section{RELATED WORK}

Microgrid is a new grid structure to group DRERs and local residential loads. In [4], the authors review the MG structure with distributed energy resources. In [23], the integration of random wind power generation into the power grid for cost effective operation is investigated. In [24], the authors propose a useful online method to discover all available DRERs within the microgrid in the islanded mode and compute a DRER access strategy. The problem of optimal residential demand management is studied in [25], aiming to adapt to time-varying energy generation and prices, and maximize user benefit. In [26], the authors investigate energy storage management with a dynamic programming approach. The size of the ESSs for MG energy storage is explored in [27].

Stochastic optimization is an important mathematical tool for resource allocation. In [28], three stochastic approximation methods are proposed to compute the optimal base-stock levels. The iteratively converged optimality is proved. Lyapunov optimization is a useful stochastic optimization method [11]. It integrates the Lyapunov stability concept of control theory with optimization and provides an efficient framework for solving schedule and control problems. It has been widely used and extended in the communications and networking areas [11], [12]. In two recent work [13], [29], the Lyapunov optimization method is applied to jointly optimize power procurement and dynamic pricing. In [13], the authors investigate the problem of profit maximization for delay tolerant consumers. In [29], the authors study electricity storage management for data centers, aiming to meet the workload requirement. Both of the work are designed based on a single energy consumption entity model.

In this paper, we investigate a novel smart energy management system for MGs based on the concept of QoSE, which is different from above work. By jointly considering multiple residents, ESSs and utility market participation, the adaptive electricity scheduling policy is designed with Lyapunov optimization for ensuring the quality of service of the electricity usage and minimizing the MG operation cost. This work is inspired by the prior work on Lyapunov optimization and in particular, the work that utilize this technique for renewable energy allocation to delay tolerant consumers [13]. Some of the proofs follow the generic Lyapunov optimization approach, such as defining the Lyapunov drift and the drift-plus-penalty method [12].

\section{CONCLUSION}

In this paper, we developed an online adaptive electricity scheduling algorithm for smart energy management in MGs by jointly considering renewable energy penetration, ESS management, residential demand management, and utility market participation. We introduced a QoSE model by taking into account minimization of the MG operation cost, while maintaining the outage probabilities of resident quality usage. We transformed the QoSE control problem and ESS management problem into queue stability problems by introducing the QoSE virtual queues and battery virtual queues. The Lyapunov optimization method was applied to solve the problem with an efficient online electricity scheduling algorithm, which has deterministic performance bounds. Our simulation study validated the superior performance of the proposed approach.

In this work, we focused on the microgrid operation on the energy market and the energy scheduling in the MG, but did not consider the daily pattern of electricity demand and price. It would be interesting to exploit such daily pattern to extend the proposed algorithm by modeling the interaction between delayed requests and future prices under the demand response strategy in the future work. 
APPENDIX A

DERIVATION OF EQUATION (18)

With the drift defined as in (17), we have

$$
\begin{aligned}
\Delta(\vec{\Theta}(t))= & \frac{1}{2} \mathbb{E}\left\{\sum_{k=1}^{K}\left[\left(X_{k}(t+1)\right)^{2}-\left(X_{k}(t)\right)^{2} \mid X_{k}(t)\right]\right. \\
& \left.+\sum_{n=1}^{N}\left[\left(Z_{n}(t+1)\right)^{2}-\left(Z_{n}(t)\right)^{2} \mid Z_{n}(t)\right]\right\} \\
\leq & \frac{1}{2} \mathbb{E}\left\{\sum _ { k = 1 } ^ { K } \left[\left(D_{k}(t)\right)^{2}+\left(R_{k}(t)\right)^{2}\right.\right. \\
& +\frac{1}{2} \mathbb{E}\left\{\sum _ { n = 1 } ^ { N } \left[X_{k}(t)\left(R_{k}(t)-D^{2}+\left(\delta_{n}(t)\right) \mid X_{n}(t)\right)^{2}\right.\right. \\
& \left.\left.+2 Z_{n}(t)\left(I_{n}(t)-\delta_{n} \alpha_{n}(t)\right) \mid Z_{n}(t)\right]\right\} \\
& \frac{1}{2} \sum_{k=1}^{K} \mathbb{E}\left\{\left[\left(D_{k}(t)\right)^{2}+\left(R_{k}(t)\right)^{2}\right]\right\} \\
& +\sum_{k=1}^{K} \mathbb{E}\left\{X_{k}(t)\left(R_{k}(t)-D_{k}(t)\right) \mid X_{k}(t)\right\} \\
& +\frac{1}{2} \sum_{n=1}^{N} \mathbb{E}\left\{\left[\left(1+\left(\delta_{n}\right)^{2}\right)\left(\alpha_{n}(t)\right)^{2}+\left(p_{n}(t)\right)^{2}\right]\right\} \\
& +\frac{1}{2} \sum_{n=1}^{N} \mathbb{E}\left\{2 Z_{n}(t)\left(1-\delta_{n}\right) \alpha_{n}(t)\right. \\
& +\sum_{k=1}^{N} \mathbb{E}\left\{X_{k}(t)\left(R_{k}(t)-D_{k}(t)\right) \mid X_{k}(t)\right\} \\
\leq & \left.B+\sum_{n=1}^{N} \mathbb{E}\left\{Z_{n}(t)+\alpha_{n}(t)\right) p_{n}(t) \mid Z_{n}(t)\right\} \\
& \left.\left.-\delta_{n}\right) \alpha_{n}(t) \mid Z_{n}(t)\right\} \\
& \\
& \\
&
\end{aligned}
$$

where

$B=(1 / 2) \sum_{k=1}^{K}\left(\max \left\{D_{k}^{\max }, R_{k}^{\max }\right\}\right)^{2}+(1 / 2) \sum_{n=1}^{N}(2+$ $\left.\delta_{n}^{2}\right)\left(\alpha_{n}^{\max }\right)^{2}$ is a constant.

\section{APPENDIX B}

PROOF OF LEMMA 1

Proof: In part 1) of Lemma 1, if $Q(t)>0$, we have $S(t)=0$ according to (7). The objective function of problem (21) becomes $V Q(t) C(t)+\sum_{k=1}^{K} X_{k}(t)\left(R_{k}(t)-\right.$ $\left.D_{k}(t)\right)-\sum_{n=1}^{N}\left(Z_{n}(t)+\alpha_{n}(t)\right) p_{n}(t)$.

We first prove Lemma 1-1a). If $X_{k}(t)>-V C(t)$, we assume $R_{k}(t)>0$. Then we have $D_{k}(t)=0$ according to (4). Accordingly, the object function is transformed to

$$
\begin{aligned}
V Q(t) C(t) & +\sum_{i \neq k} X_{i}(t)\left(R_{i}(t)-D_{i}(t)\right) \\
- & \sum_{n=1}^{N}\left(Z_{n}(t)+\alpha_{n}(t)\right) p_{n}(t)+X_{k}(t) R_{k}(t)
\end{aligned}
$$

$$
\begin{aligned}
> & V Q(t) C(t)+\sum_{i \neq k} X_{i}(t)\left(R_{i}(t)-D_{i}(t)\right) \\
& -\sum_{n=1}^{N}\left(Z_{n}(t)+\alpha_{n}(t)\right) p_{n}(t)-V C(t)(P(t)+Q(t) \\
& -\sum_{i \neq k}\left(R_{i}(t)-D_{i}(t)\right)-\sum_{n=1}^{N} p_{n}(t) \\
= & V\left[\sum_{i \neq k}\left(R_{i}(t)-D_{i}(t)\right)+\sum_{n=1}^{N} p_{n}(t)-P(t)\right] C(t) \\
& +\sum_{i \neq k} X_{i}(t)\left(R_{i}(t)-D_{i}(t)\right) \\
& -\sum_{n=1}^{N}\left(Z_{n}(t)+\alpha_{n}(t)\right) p_{n}(t) .
\end{aligned}
$$

The above inequality is due to $X_{k}(t)>-V C(t)$ and $R_{k}(t)=$ $P(t)+Q(t)-\sum_{i \neq k}\left(R_{i}(t)-D_{i}(t)\right)-\sum_{n=1}^{N} p_{n}(t) \geq 0$. The last expression shows, given the assumption $R_{k}(t)>0$, we may find another feasible electricity allocation scheme $\tilde{Q}(t)=$ $\sum_{i \neq k}\left(R_{i}(t)-D_{i}(t)\right)+\sum_{n=1}^{N} p_{n}(t)-P(t)$, which can achieve a smaller objective value by choosing $R_{k}(t)=0$ and $D_{k}(t)=$ 0 . This contradicts with the assumption $R_{k}(t)>0$. Thus, we prove that $R_{k}(t)=0$ when $X_{k}(t)>-V C(t)$, under the situation $Q(t)>0, S(t)=0$.

We then prove the second part of Lemma 1-1a). It follows (4) that $R_{k}(t)=0$ if $D_{k}(t)>0$. Then the objective function becomes

$$
\begin{aligned}
& V Q(t) C(t)+\sum_{i \neq k} X_{i}(t)\left(R_{i}(t)-D_{i}(t)\right) \\
& -\sum_{n=1}^{N}\left(Z_{n}(t)+\alpha_{n}(t)\right) p_{n}(t)-X_{k}(t) D_{k}(t) \\
& >V Q(t) C(t)+\sum_{i \neq k} X_{i}(t)\left(R_{i}(t)-D_{i}(t)\right) \\
& -\sum_{n=1}^{N}\left(Z_{n}(t)+\alpha_{n}(t)\right) p_{n}(t) \\
& +V C(t)\left(\sum_{n=1}^{N} p_{n}(t)-P(t)-Q(t)+\sum_{i \neq k}\left(R_{i}(t)-D_{i}(t)\right)\right) \\
& =V\left[\sum_{i \neq k}\left(R_{i}(t)-D_{i}(t)\right)+\sum_{n=1}^{N} p_{n}(t)-P(t)\right] C(t) \\
& +\sum_{i \neq k}^{N} X_{i}(t)\left(R_{i}(t)-D_{i}(t)\right) \\
& \quad-\sum_{n=1}^{N}\left(Z_{n}(t)+\alpha_{n}(t)\right) p_{n}(t) .
\end{aligned}
$$

The above inequality is due to $X_{k}(t)<-V C(t)<0$ and $D_{i}(t)=-P(t)-Q(t)+\sum_{i \neq k}\left(R_{i}(t)+\right.$ $\left.D_{k}(t)\right)+\sum_{n=1}^{N} p_{n}(t) \quad>\quad 0$. The last expression shows, given the assumption $D_{k}(t)>0$, we may find another electricity allocation scheme with 
$\tilde{Q}(t)=\sum_{i \neq k}\left(R_{i}(t)-D_{i}(t)\right)+\sum_{n=1}^{N} p_{n}(t)-P(t)$, which can achieve a smaller objective value by choosing $R_{k}(t)=0$ and $D_{k}(t)=0$. This contradicts with the assumption $D_{k}(t)>0$. We thus prove that $D_{k}(t)=0$ when $X_{k}(t)<-V C(t)$, under the situation $Q(t)>0, S(t)=0$, which completes the proof of Lemma 1-1a).

We next prove Lemma 1-1b). For the first part, if $Z_{n}(t)>$ $V C(t)-\alpha_{n}(t)$, we assume $0 \leq p_{n}(t)<\left(1-\delta_{n}\right) \alpha_{n}(t)$. Following (20) and $S(t)=0$, we have

$$
\begin{aligned}
B+ & V Q(t) C(t)+\sum_{k=1}^{K} X_{k}(t)\left(R_{k}(t)-D_{k}(t)\right) \\
& +\sum_{j \neq n}\left(Z_{j}(t)\left(1-\delta_{j}\right) \alpha_{j}(t)-\left(Z_{j}(t)+\alpha_{j}(t)\right) p_{j}(t)\right) \\
& +Z_{n}(t)\left(1-\delta_{n}\right) \alpha_{n}(t)-\left(Z_{n}(t)+\alpha_{n}(t)\right) p_{n}(t) \\
= & B+V Q(t) C(t)+\sum_{k=1}^{K} X_{k}(t)\left(R_{k}(t)-D_{k}(t)\right) \\
& +\sum_{j \neq n}\left(Z_{j}(t)\left(1-\delta_{j}\right) \alpha_{j}(t)-\left(Z_{j}(t)+\alpha_{j}(t)\right) p_{j}(t)\right) \\
& +Z_{n}(t)\left[\left(1-\delta_{n}\right) \alpha_{n}(t)-p_{n}(t)\right]-\alpha_{n}(t) p_{n}(t) \\
> & B+V Q(t) C(t)+\sum_{k=1}^{K} X_{k}(t)\left(R_{k}(t)-D_{k}(t)\right) \\
& +\sum_{j \neq n}\left(Z_{j}(t)\left(1-\delta_{j}\right) \alpha_{j}(t)-\left(Z_{j}(t)+\alpha_{j}(t)\right) p_{j}(t)\right) \\
& +\left(V C(t)-\alpha_{n}(t)\right)\left[\left(1-\delta_{n}\right) \alpha_{n}(t)-p_{n}(t)\right] \\
& -\alpha_{n}(t) p_{n}(t) \\
= & B+V\left[\sum_{k=1}^{K}\left(R_{k}(t)-D_{k}(t)\right)+\sum_{j \neq n} p_{j}(t)-P(t)\right. \\
& \left.+\left(1-\delta_{n}\right) \alpha_{n}(t)\right] C(t) \\
& +\sum_{k=1}^{K} X_{k}(t)\left(R_{k}(t)-D_{k}(t)\right) \\
& +\sum_{j \neq n}\left(Z_{j}(t)\left(1-\delta_{j}\right) \alpha_{j}(t)-\left(Z_{j}(t)+\alpha_{j}(t)\right) p_{j}(t)\right) \\
& +Z_{n}(t)\left(1-\delta_{n}\right) \alpha_{n}(t) \\
& -\left(Z_{n}(t)+\alpha_{n}(t)\right)\left(1-\delta_{n}\right) \alpha_{n}(t) . \\
& +V \text {. }
\end{aligned}
$$

The above inequality is due to $Z_{n}(t)>V C(t)-\alpha_{n}(t)$ and the assumption $p_{n}(t)<\left(1-\delta_{n}\right) \alpha_{n}(t)$. The last equality shows, given the assumption $p_{n}(t)<\left(1-\delta_{n}\right) \alpha_{n}(t)$, we may find another electricity allocation scheme with $p_{n}(t)=\left(1-\delta_{n}\right) \alpha_{n}(t)$ and $\tilde{Q}(t)=\sum_{k=1}^{K}\left(R_{k}(t)-D_{k}(t)\right)+\sum_{j \neq n} p_{j}(t)-P(t)+(1-$ $\left.\delta_{n}\right) \alpha_{n}(t)$, which can achieve a smaller objective value. This contradicts with the previous assumption. Thus, we have $p_{n}(t) \geq\left(1-\delta_{n}\right) \alpha_{n}(t)$.

For the second part of Lemma 1-1b), assume $p_{n}(t)>0$ for $0 \leq Z_{n}(t)<V C(t)-\alpha_{n}(t)$. It follows (7) that $S(t)=0$. The objective function (20) can be written as

$$
B+V Q(t) C(t)+\sum_{k=1}^{K} X_{k}(t)\left(R_{k}(t)-D_{k}(t)\right)
$$

$$
\begin{aligned}
& +\sum_{j \neq n}\left(Z_{j}(t)\left(1-\delta_{j}\right) \alpha_{j}(t)-\left(Z_{j}(t)+\alpha_{j}(t)\right) p_{j}(t)\right) \\
& +Z_{n}(t)\left(1-\delta_{n}\right) \alpha_{n}(t)-\left(Z_{n}(t)+\alpha_{n}(t)\right) p_{n}(t) \\
> & B+V Q(t) C(t)+\sum_{k=1}^{K} X_{k}(t)\left(R_{k}(t)-D_{k}(t)\right) \\
& +\sum_{j \neq n}\left(Z_{j}(t)\left(1-\delta_{j}\right) \alpha_{j}(t)-\left(Z_{j}(t)+\alpha_{j}(t)\right) p_{j}(t)\right) \\
& +Z_{n}(t)\left(1-\delta_{n}\right) \alpha_{n}(t)-V C(t) p_{n}(t) \\
\geq & B+V\left[-P(t)+\sum_{k=1}^{K}\left(R_{k}(t)-D_{k}(t)\right)+\sum_{j \neq n} p_{j}(t)\right] C(t) \\
& +\sum_{k=1}^{K} X_{k}(t)\left(R_{k}(t)-D_{k}(t)\right) \\
& +\sum_{j \neq n}\left(Z_{j}(t)\left(1-\delta_{j}\right) \alpha_{j}(t)\right) \\
& -\sum_{j \neq n}\left(\left(Z_{j}(t)+\alpha_{j}(t)\right) p_{j}(t)\right) .
\end{aligned}
$$

The first inequality is due to $0 \leq Z_{n}(t)<V C(t)-\alpha_{n}(t)$ and the assumption $p_{n}(t)>0$. The second inequality is due to the non-negativity of $Z_{n}(t)$ and $\alpha_{n}(t)$. The last equation shows, given the assumption $p_{n}(t)>0$, we may find another electricity allocation scheme with $p_{n}(t)=0$ and $\tilde{Q}(t)=-P(t)+$ $\sum_{k=1}^{K}\left(R_{k}(t)-D_{k}(t)\right)+\sum_{j \neq n} p_{j}(t)$, which can achieve a smaller objective value. This contradicts with the previous assumption. Thus, we have $p_{n}(t)=0$, which completes the proof of Lemma 1-1b).

In part 2) of Lemma 1 , if $S(t)>0$, we have $Q(t)=0$ according to (7). The objective function (21) becomes $-V S(t) W(t)+\sum_{k=1}^{K} X_{k}(t)\left(R_{k}(t)-D_{k}(t)\right)-\sum_{n=1}^{N}\left(Z_{n}(t)+\right.$ $\left.\alpha_{n}(t)\right) p_{n}(t)$. We can prove part 2) with a similar approach as in the case of part 1 . The detailed proof is omitted for brevity.

\section{APPENDIX C \\ Proof OF LEMMA 2}

Proof: Since $0 \leq C_{\min } \leq C(t) \leq C_{\max }$ and $V>0$, we have $R_{k}(t)=0$ when $X_{k}(t)<-V C_{\max }$, and $D_{k}(t)=0$ when $X_{k}(t)>-V C_{\min }$ according to Lemma 1-1). Similarly, since $0 \leq W_{\min } \leq W(t) \leq W_{\max }$ and $V>0$, we obtain $R_{k}(t)=$ 0 when $X_{k}(t)<-V W_{\max }$, and $D_{k}(t)=0$ when $X_{k}(t)>$ $-V W_{\min }$ according to Lemma 1-2)

Since $C_{\max }>W_{\max }$ and $C_{\min }>W_{\min }$, we conclude that if $X_{k}(t)>-V W_{\min }$, the optimal solution always select $R_{k}(t)=0$. If $X_{k}(t)<-V C_{\max }$, the optimal solution always select $D_{k}(t)=0$. The proof is completed.

\section{APPENDIX D}

\section{PROOF OF LEMMA 3}

Proof: The proof directly follows Lemma 1 and is similar to the proof of Lemma 2. We omit the details for brevity. 


\section{REFERENCES}

[1] Y. Huang, S. Mao, and R. M. Nelms, "Adaptive electricity scheduling in microgrids,” in Proc. IEEE INFOCOM’13, Turin, Italy, Apr. 2013, pp. 1-9.

[2] "Battery and electric vehicle report," Jul. 2010 [Online]. Available: http://www.whitehouse.gov/files/documents/Battery-and-Electric-Vehicle-Report-FINAL.pdf

[3] X. Fang, S. Misra, G. Xue, and D. Yang, "Smart grid-The new and improved power grid: A survey," IEEE Commun. Surveys Tuts., vol. PP, no. 99, pp. 1-37, Dec. 2011.

[4] J. Huang, C. Jiang, and R. Xu, "A review on distributed energy resources and microgrid," Renewable Sustain. Energy Rev., vol. 12, no. 9, pp. 2472-2483, Dec. 2008.

[5] H. Farhangi, "The path of the smart grid," IEEE Power Energy Mag., vol. 8, no. 1, pp. 18-28, Jan.-Feb. 2010.

[6] S. Shao, M. Pipattanasomporn, and S. Rahman, "Demand response as a load shaping tool in an intelligent grid with electric vehicles," IEEE Trans. Smart Grid, vol. 2, no. 4, pp. 624-631, Dec. 2011.

[7] Y. Huang and S. Mao, "On Quality of Usage provisioning for electricity scheduling in microgrids," IEEE Syst. J., 10.1109/JSYST.2013. 2260941, to be published.

[8] E. Fumagalli, J. W. Black, I. Vogelsang, and M. Ilic, "Quality of service provision in electric power distribution systems through reliability insurance," IEEE Trans. Power Syst., vol. 19, no. 3, pp. 1286-1293, Aug. 2004.

[9] Y. Wang, S. Mao, and R. M. Nelms, "An online algorithm for optimal real-time energy distribution in smart grid," IEEE Trans. Emerging Topics Comput., vol. 1, no. 1, pp. 10-21, Jul. 2013.

[10] Y. Wang, S. Mao, and R. M. Nelms, "A distributed online algorithm for optimal real-time energy distribution in smart grid," in Proc. IEEE GLOBECOM 2013, Atlanta, GA, USA, Dec. 2013.

[11] L. Tassiulas and A. Ephremides, "Stability properties of constrained queueing systems and scheduling policies for maximum throughput in multihop radio networks," IEEE Trans. Autom. Control, vol. 37, no. 12, pp. 1936-1948, Dec. 1992

[12] M. Neely, E. Modiano, and C. Rohrs, "Dynamic power allocation and routing for time-varying wireless networks," IEEE J. Sel. Areas Commun., vol. 23, no. 1, pp. 89-103, Jan. 2005.

[13] M. J. Neely, A. S. Tehrani, and A. G. Dimakis, "Efficient algorithms for renewable energy allocation to delay tolerant consumers," in Proc. IEEE SmartGridComm'10, Oct. 2010, pp. 549-554.

[14] J. Slotine and W. Li, Applied Nonlinear Control. Englewood Cliffs, NJ, USA: Prentice-Hall, 1991.

[15] L. Georgiadis, M. J. Neely, and L. Tassiulas, "Resource allocation and cross-layer control in wireless networks," Found. Trends Netw., vol. 1, no. 1, pp. 1-144, 2006

[16] M. J. Neely, Stochastic Network Optimization With Application to Communication and Queueing Systems. San Rafael, CA, USA: Morgan \& Claypool, 2010.

[17] T. T. Kim and H. Poor, "Scheduling power consumption with price uncertainty," IEEE Trans. Smart Grid, vol. 2, no. 3, pp. 519-527, Sep. 2011.

[18] M. J. Neely, "Stock market trading via stochastic network optimization," in Proc. IEEE CDC'10, Atlanta, GA, USA, Dec. 2010, pp. 1-8.

[19] M. J. Neely and R. Urgaonkar, "Opportunism, backpressure, and stochastic optimization with the wireless broadcast advantage," in Proc. Asilomar Conf. Signals, Syst., Comput. '08, Pacific Grove, CA, USA, Oct. 2008, pp. 1-7.

[20] National Renewable Energy Laboratory, "Western Wind Resources Dataset" [Online]. Available: http://wind.nrel.gov/Web_nrel/

[21] S. B. Peterson, J. F. Whitacre, and J. Apt, "The economics of using plug-in hybrid electric vehicle battery packs for grid storage," J. Power Sources, vol. 195, no. 8, pp. 2377-2384, 2010.

[22] Electric Reliability Council of Texas [Online]. Available: http://www. ercot.com/

[23] X. Liu, "Economic load dispatch constrained by wind power availability: A wait-and-see approach," IEEE Smart Grid, vol. 1, no. 3, pp. 347-355, Dec. 2010.

[24] X. Fang, D. Yang, and G. Xue, "Online strategizing distributed renewable energy resource access in islanded microgrids," in Proc.IEEE GLOBECOM'11, Huston, TX, USA, Dec. 2011, pp. 1931-1937.
[25] N. Li, L. Chen, and S. H. Low, "Optimal demand response based on utility maximization in power networks," in Proc. IEEE PES Gen. Meet., Detroit, MI, USA, Jul. 2011, pp. 1-8.

[26] I. Koutsopoulos, V. Hatzi, and L. Tassiulas, "Optimal energy storage control policies for the smart power grid," in Proc. IEEE SmartGridComm'11, Oct. 2011, pp. 475-480.

[27] S. X. Chen, H. B. Gooi, and M. Q. Wang, "Sizing of energy storage for microgrids," IEEE Trans. Smart Grid, vol. 3, no. 1, pp. 142-151, Mar 2012 .

[28] S. Kunnumkal and H. Topaloglu, "Using stochastic approximation methods to compute optimal base-stock levels in inventory control problems," Oper. Res., vol. 56, no. 3, pp. 646-664, May-Jun. 2008.

[29] R. Urgaonkar, B. Urgaonkar, M. J. Neely, and A. Sivasubramaniam, "Optimal power cost management using stroed energy in data centers," in Proc. ACM SIGMETRICS'11, San Jose, CA, USA, Jun. 2011, pp. 221-232.

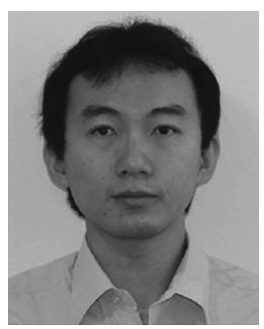

Yingsong Huang (S'12) received the B.S. degree in automation and the M.S. degree in control theory and control engineering from Chongqing University, Chongqing, China. He received the Ph.D. in electrical and computer engineering from Auburn University, Auburn, AL, USA, in May 2013.

He was a Senior R\&D Engineer and Vice Supervisor with Advantech Co. Ltd., Beijing, China, and Taipei, Taiwan, from July 2003 to July 2007. Currently he is a Software Engineer at NetApp, Pittsburgh, PA, USA. His research interests include modeling, control and optimization in microgrids, smart grid and computer networks.

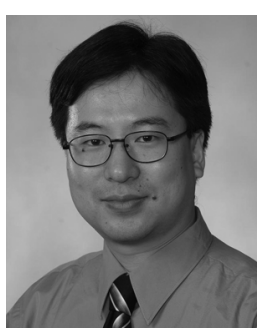

Shiwen Mao (S'99-M'04-SM'09) received the $\mathrm{Ph} . \mathrm{D}$. in electrical and computer engineering from Polytechnic University, Brooklyn, NY, USA.

Currently, he is the McWane Associate Professor in the Department of Electrical and Computer Engineering, Auburn University, Auburn, AL, USA. His research interests include wireless networks and multimedia communications, with current focus on cognitive radio, small cells, $60 \mathrm{GHz}$ mmWave networks, free space optical networks, and smart grid.

Dr. Mao is on the Editorial Board of IEEE TRANSACTIONS ON WIRELESS COMMUNICATIONS, IEEE Internet of Things Journal, IEEE Communications Surveys and Tutorials, Elsevier Ad Hoc Networks Journal, and Wiley International Journal on Communication Systems. He received the 2013 IEEE ComSoc MMTC Outstanding Leadership Award and the NSF CAREER Award in 2010. He is a co-recipient of The IEEE ICC 2013 Best Paper Award and The 2004 IEEE Communications Society Leonard G. Abraham Prize in the Field of Communications Systems.

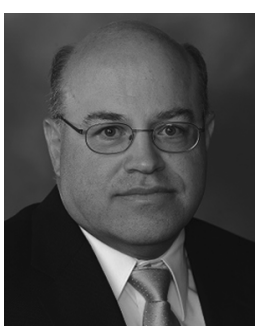

R. M. Nelms (F'04) received the B.E.E. and M.S. degrees in electrical engineering from Auburn University, Auburn, AL, USA, in 1980 and 1982, respectively. He received the Ph.D. degree in electrical engineering from Virginia Polytechnic Institute and State University, Blacksburg, VA, USA, in 1987.

$\mathrm{He}$ is currently Professor and Chair of the Department of Electrical and Computer Engineering at Auburn University. His research interests are in power electronics, power systems, and electric machinery.

Dr. Nelms was named an IEEE Fellow in 2004 "for technical leadership and contributions to applied power electronics.” He is a Registered Professional Engineer in Alabama. 